\title{
Preventing cervical cancer
}

\section{South African women with cervical cancer often present late and have}

\section{a poor prognosis as a result.}

\section{MH (HENNIE) BOTHA, MB ChB, FCOG, MMed \\ Gynaecological Oncologist, Unit for Gynaecologic Oncology, Department of Obstetrics and Gynaecology, Stellenbosch University and Tygerberg Hospital}

Hennie Botha is a registered gynaecological oncologist. He has a research interest in fertility preservation in female cancer patients. He is a full-time clinician and teaches under- and postgraduate students.

E-mail:mhbotha@sun.ac.za

Cervical cancer remains the most common cancer diagnosed in women in southern Africa, with an estimated lifetime risk of 1 in 26. ${ }^{1}$ Unfortunately most of these cancers are also diagnosed at a late stage, with subsequent poor prognosis for long-term survival. This very high incidence is particularly sad in an era where advances in medical science have made cervical cancer a mostly preventable disease. In most developed economies cervical cancer has become a less common occurrence due to intensive screening and preventive strategies.

\section{In most developed economies cervical cancer has become a less common occurrence due to intensive screening and preventive strategies.}

We are entering an exciting new era in the fight against cervical cancer with new vaccine technology becoming available. Vaccines against oncogenic (cancer causing) strains of human papillomavirus (HPV) will hopefully reduce cervical cancer rates globally even further and provide hope for South Africa. Professor Harald Zur Hausen was awarded the Nobel Prize for physiology and medicine in 2008 for his very important work on papillomaviruses and cervical carcinogenesis. ${ }^{2}$ This highlights the progress that has been made over the last few years in terms of the understanding of cervical cancer aetiology and new strategies for prevention.

\section{Aetiology of cervical cancer}

Persistent infection with an oncogenic strain of HPV is a necessary risk factor for the development of cervical cancer. ${ }^{3}$ Oncogenic types of HPV have the ability to integrate certain parts of the viral DNA into the human genome. The oncoproteins E6 and E7 inactivate very important processes associated with tumour-suppressor genes like P53 and PRb gene functions. Low-risk HPV infection is associated with warts on the epithelial surfaces of the genital area but also warts on hands and feet. It is important to recognise that low-risk HPV types causing plantar warts and warts on the skin are not associated with cancer but belong to the same family of papillomaviruses. HPV is highly infectious and 'most sexually active people will get HPV at some time in their lives', making it a very common sexually transmitted infection. ${ }^{4}$ This ubiquitous infection does not cause disease in all the infected individuals and certain processes make individuals more susceptible to the formation of pre-malignant and malignant disease. Because
HPV is mostly an epithelial disease the virus is poorly presented to the adaptive immune system, which is important for induction of long-term immunity. Most natural infections of HPV do not cause significant immunoglobulin responses and therefore the immune response after natural infections is not very pronounced. ${ }^{5}$ After initial exposure to HPV there is an incubation period of 1 - 8 months, after which the first HPV-related lesions might appear. There is active growth of the virus for a period of $3-6$ months but usually there are host-immune responses that will, in most cases, clear the infection by about 9 months. A large percentage of the population will have sustained clinical remission but a small proportion will develop resistant or recurrent disease and become HPV-DNA positive on repeated testing. These are the individuals who will be at highest risk for the development of pre-malignant conditions and later invasive cancer. We now know that changes in cells due to HPV infection of the cervix are the first step in a series of slow changes that can lead to cancer years later.

\section{Persistent infection with an oncogenic strain of HPV is a necessary risk factor for the development of cervical cancer.}

\author{
Prevention of disease \\ Disease prevention strategies can be categorised into primary \\ prevention, secondary prevention and tertiary prevention.
}

Primary prevention aims to reduce the risk of an individual getting a particular disease by reducing the disease-causing agents in the environment. In the case of cervical cancer the most important aetiological factor for the development of pre-malignant and malignant disease is persistent infection with oncogenic types of HPV infection. By reducing the risk for HPV infection the incidence of cervical cancer should decrease drastically. These may include measures like education about safe sexual practices including mutual monogamy and barrier contraceptive use (although this is not fully protective against HPV infection). Recently vaccination against HPV infection has become available in South Africa. The very strong association between HIV infection with papillomavirus-associated disease also gives the opportunity for primary prevention by reducing HIV seropositivity. Other risk factors include smoking, long-term use of oral contraceptives and high parity. 
Secondary prevention of cervical cancer involves screening for early diagnosis and treatment of pre-cancerous lesions. Secondary screening methods are described below.

Tertiary prevention of disease aims to downstage the disease. When detecting the disease at an earlier stage, simpler forms of treatment may still be given successfully. A good example is visual inspection with acetic acid of the cervix to identify early invasive cancers or pre-malignant conditions. This strategy was tested in a large study from India and it was shown that it can reduce the mortality rate due to cervical cancer by approximately $35 \%{ }^{6}$

\section{Primary prevention: human papillomavirus vaccines}

Over the last 20 years scientists have developed a progressively better understanding of the pathogenesis of HPV infection and cervical cancer. This has led to the development of vaccines against HPV infection. ${ }^{2}$ Virus-like particles (VLPs) that do not contain any virus DNA have been produced in the laboratory. These VLPs, when injected into the human circulation, elicit a strong immune response. These vaccines have produced fantastic results in terms of protection against disease and persistent infection and are now widely used in many different parts of the world. ${ }^{7,8}$ The VLP vaccines are very safe and have been extensively studied in large populations. Currently there are two registered vaccines for HPV vaccination, namely Cervarix containing HPV types 16 and 18 and Gardasil, which protects against HPV 16 and 18 and also two low-risk types, numbers 6 and 11 . HPV 6 and 11 are the most common viruses causing genital warts. Both vaccines have been studied in large populations and have been found to be safe, effective and well tolerated. The virus-like particles are combined with an immune stimulant called an adjuvant which leads to improved immunoglobulin production against the vaccine types of VLPs. It has been shown recently that the vaccines protect against more types of HPV infection than just those that are present in the vaccines. ${ }^{9}$ Cervarix has been shown to have excellent crossprotection against types 31 and 45 and Gardasil has shown partial cross-protection against types $31,33,45,52$ and 58. At present the exact period of protection is not known, but longitudinal studies over more than 7 years have shown that these vaccines will most likely be protective for a very long period of time and a booster should not be necessary. Both vaccines have a 3-dose schedule, with the second dose after 1 or 2 months and a third dose after 6 months.

\begin{tabular}{|c|c|c|}
\hline Vaccine & Gardasil & Cervarix \\
\hline HPV types & $6,11,16,18$ & 16,18 \\
\hline Dosing schedule & $0,2,6$ months & $0,1,6$ months \\
\hline Adjuvant & $\begin{array}{l}\text { AAHS (amorphous } \\
\text { aluminum hydroxyphosphate } \\
\text { sulfate) }\end{array}$ & $\begin{array}{l}\mathrm{ASO}_{4} \text { (aluminum } \\
\text { hydroxide + MPL) }\end{array}$ \\
\hline $\begin{array}{l}\text { VLP preparation } \\
\text { system }\end{array}$ & Yeast & Insect cell \\
\hline $\begin{array}{l}\text { Efficacy against disease } \\
\text { caused by HPV types in } \\
\text { the vaccine }\end{array}$ & $100 \%^{11}$ & $98.1 \%^{8}$ \\
\hline
\end{tabular}

In order for the vaccine to be effective in preventing HPV infection, it must be given before exposure to HPV. This is of course before exposure to sexual contact. There may be some differences between populations, but in general most authorities feel that girls around the age of 11 - 12 years, just before leaving primary school, may be the most suitable for mass vaccination.

\section{Most natural infections of HPV do not cause significant immunoglobulin responses and therefore the immune response after natural infections is not very pronounced.}

High vaccine coverage is needed if significant improvement of cervical cancer rates is to be achieved. In many First-World countries the introduction of the vaccines has been rapid and very well organised. Examples of well-organised vaccination programmes for girls are the National Health Service in the UK and the Australian government programme. Both these programmes aim to vaccinate all girls around the age of 12 but for the first few years also include a catch-up group of slightly older women. At present most national or regional vaccination programmes do not include boys, but it makes sense to include them when the vaccines become less expensive. ${ }^{10}$ The uptake of these vaccines has been good, with an acceptance rate of over $80 \%$ in both Australia and the UK. In South Africa Gardasil and Cervarix are both available in the private sector and are currently being considered for a national vaccination programme. A vaccination programme in
South Africa will almost certainly make a significant difference in the cervical cancer incidence within a few years. South Africa has a high rate of infant vaccination and the principles of vaccination are well known to health care professionals. The benefit over the medium to long term will be enormous in terms of cost of treatment and diagnosis of pre-malignant and malignant cervical disease. The commercially available vaccines are listed in Table I.

\section{secondary prevention of cervical cancer}

\section{Cervical cytology}

George Papanicolaou initially described the examination of posterior vaginal fornix cells to detect precursors for cervical cancer in 1928. A subsequent publication in 1941 was one of the most important articles in the history of cancer screening. Since then few modifications were introduced. Ayre, in 1947, introduced the wooden spatula. Cervical cytology (Pap test) remains a highly effective and trusted screening tool for cervical cancer. Regular cytology smears, combined with treatment of premalignant lesions, can reduce the incidence of cervical cancer drastically if it is used on a high proportion of the entire population. Opportunistic screening may be effective in certain populations where patient education and motivation are high, but for a programme to work well a populationbased screening policy is more effective. In certain parts of South Africa (particularly in the private sector) opportunistic screening with regular Pap smears has resulted in a significant drop in the rate of cervical cancer. The national policy for cervical cancer screening in the public sector was introduced in 2000 by the Department of Health. With this programme every woman in South Africa should have three cytology smears 10 years apart, starting at the age of 30. This is a good model and compares well with the World Health Organization suggestion of a single screen per woman per lifetime in low-income countries. However, 
in the state sector the national cytology screening programme has not been implemented widely.

Liquid-based cytology is a similar technique, where the collected cells are transported in liquid to the laboratory. This method of preparation of cervical cytology may decrease the false-negative rate of standard Pap smears. Liquid-based cytology uses the same principle as the Pap smear but the technique may have a slightly better sensitivity and specificity for the diagnosis of high-grade intra-epithelial lesions.

\section{By reducing the risk for HPV infection the incidence of cervical cancer should decrease drastically.}

The term now used in cytological screening of Pap smears is 'squamous intraepithelial lesion' (SIL).

Low-grade squamous intraepithelial lesion (LSIL) refers to early changes in the size, shape and nuclei of cells. Most low-grade lesions clear on their own. However, with time, others may become more abnormal, forming a high-grade lesion. It is usually not necessary to treat or investigate further when LSIL is present on a cytology smear. This is however an indication that the test should be repeated a few months later and if the LSIL is still present on the repeat it will need further investigation.

High-grade squamous intraepithelial lesion (HSIL) means there are a larger number of more abnormal pre-cancerous cells. In the past, these lesions were referred to as moderate or severe dysplasia, or carcinoma in situ. When HSIL is found on a Pap smear further tests like a colposcopy or Lletz are necessary.

If intraepithelial neoplastic cells break through the basement membrane, the disease is regarded as invasive cervical cancer.

\section{Human papillomavirus DNA testing}

Recently technology for the testing of specific human papillomavirus types has improved dramatically. The newest generation of HPV tests is very accurate and will detect very low numbers of virus particles in any given sample. Broadly there are two types of HPV tests, the first being a test for a 'basket' of high-grade viruses (usually 16, 18, 31, 33, 35, 39, 45, $51,52,56,58,59$ and 68$)$. One example is the hybrid capture II test. The other type of HPV test is called genotyping and tests for many single types of HPV. The newer tests use polymerase chain reaction (PCR) and are completely automated. Genotyping HPV tests are available at most private pathology laboratories in South Africa and are relatively expensive. The first type, the so-called 'basket test', usually gives simple yes/no answers for the positivity of high-grade HPV types. HPV genotyping however is reported on a strip where the different HPV types will create a colour development reaction if present in the sample. It is therefore possible to see the exact types of HPV present in the sample, even at very, very low concentrations.

HPV testing has a very high sensitivity for the detection of HSIL lesions. Even if the specificity is significantly lower than that of cytology, it has an excellent negative predictive value and the test can be done on dry or wet samples. It is easy to include HPV testing in a liquid-based cytology setting. In future it may even be possible that a patient may self-collect samples of HPV in the form of a self-administered vaginal swab or even a tampon.

If HPV testing is to be used as a screening tool, it is important to only test women over the age of 35 . If younger women are tested the incidence of HPV infection will be very high and the detection rate for true histological abnormality will be disappointingly low. If, however, older women test positive for HPV, the chances are that they have persistent infection with concomitant cytological abnormalities. This may become the screening test of choice in a vaccinated population. ${ }^{12}$

At present cervical cytology remains the cornerstone of cervical cancer screening. However, $4-10 \%$ of cytology smears are reported as atypical squamous cells of undetermined significance (ASCUS) that may make management difficult. HPV testing in these patients may help to triage patients for further management. There is strong evidence to support this strategy. ${ }^{13}$

\section{Visual inspection with acetic acid}

Visual inspection with acetic acid of the cervix is a method used in low-resource settings to identify early invasive cancers or pre-malignant conditions. The cervix is visualised during a speculum examination and a weak solution of acetic acid is applied to stain abnormal epithelial lesions white.
A vaccination programme in South Africa will almost certainly make a significant difference in the cervical cancer incidence within a few years.

\section{HIV and cervical cancer}

HIV infection is associated with high incidences of CIN lesions and also persistent HPV infection. ${ }^{14,15}$ An important study from Durban showed that HIV infection may cause HPV infection to progress quickly from pre-invasive disease to frank invasion. The HIV-positive population developed cervical cancer 15 years earlier than the HIV-negative control group. ${ }^{16} \mathrm{~A}$ local study has shown that HIVinfected women are also at an increased risk of pre-cancerous lesions. ${ }^{15}$ The national HIV management policy includes annual cervical cytology. However, recently this has been questioned and the Western Cape policy states that HIV-positive women should be tested every 3 years if the initial screen is normal. This is still more frequent than the national screening protocol of three cytology tests per woman per lifetime starting after the age of 30 years.

\section{References}

1. Mqoqi N, Kellett P, Sitas F, Jula M. Incidence of Histologically Diagnosed Cancer in South Africa, 1998 - 1999. Johannesburg: National Cancer Registry of South Africa, National Health Laboratory Service, 2004.

2. Zur Hausen H. Papillomaviruses in the causation of human cancers - a brief historical account. Virology 2009; 384(2): 260-265.

3. Stanley M. Prevention strategies against the human papillomavirus: the effectiveness of vaccination. Gynecol Oncol 2007; 107(2 Suppl 1): S19-23.

4. CDC. What Women with a Positive HPV Test Result Should Know (cited 2009 March). Available from http://www.cdc.gov/std/hpv/ common-clinicians/InsertPos.pdf.

5. Stanley M. Immunobiology of HPV and HPV vaccines. Gynecol Oncol 2008; 109(2 Suppl): S15-21.

6. Sankaranarayanan R, Esmy R, Rajkumar R, et al. Effect of visual screening on cervical cancer incidence and mortality in Tamil Nadu, India: a cluster-randomised trial. Lancet 2007; 370(9585): 398-406.

7. Wheeler CM, Kjaer SK, Sigurdsson K, et al. The impact of quadrivalent human papillomavirus (HPV; types $6,11,16$, and 18 ) L1 virus-like particle vaccine on infection and disease due to oncogenic nonvaccine HPV 
types in sexually active women aged 16-26 years. J Infect Dis 2009; 199(7): 936-944.

8. Paavonen J, Jenkins D, Bosch FX, et al. Efficacy of a prophylactic adjuvanted bivalent L1 virus-like-particle vaccine against infection with human papillomavirus types 16 and 18 in young women: an interim analysis of a phase III double-blind, randomised controlled trial. Lancet 2007; 369(9580): 2161-2170.

9. Ault KA. Human papillomavirus vaccines and the potential for cross-protection between related HPV types. Gynecol Oncol 2007; 107(2 Suppl 1): S31-33.

10. Kubba T. Human papillomavirus vaccination in the United Kingdom: what about boys? Reprod Health Matters 2008; 16(32): 97-103.

11. Villa LL, Costa CA, Petta RP, et al. High sustained efficary of a prophylactic quadrivalent human papillomavirus types $6 / 11 / 16 / 18$ L1 virus-like particle vaccine through 5 years of follow-up. $\mathrm{Br} \mathrm{J}$ Cancer 2006; 95(11): 1459-1466.

12. Wright TC jun. Cervical cancer screening in the 21st century: is it time to retire the PAP smear? Clin Obstet Gynecol 2007; 50(2): 313-323.
13. Arbyn M, Buntinx F, Van Ranst M, et al. Results of a randomized trial on the management of cytology interpretations of atypical squamous cells of undetermined significance. Am J Obstet Gynecol 2003; 188(6): 1383-1392.

14. Ellerbrock TV, Chiasson MA, Bush TJ, et al. Incidence of cervical squamous intraepithelial lesions in HIV-infected women. JAMA 2000; 283(8): 1031-1037.
15. Moodley JR, Hoffman M, Carrara BR, et al. HIV and pre-neoplastic and neoplastic lesions of the cervix in South Africa: a casecontrolled study. BMC Cancer 2006; 6: 135.

16. Moodley M, Moodley J, Kleinschmidt I. Invasive cervical cancer and human immunodeficiency virus (HIV) infection: a South African perspective. Int J Gynecol Cancer 2001; 11(3): 194-197.

\section{In a nutshell}

- HPV vaccines offer a unique opportunity to change the cervical cancer scene in South Africa, where secondary prevention strategies have had only limited success.

- A well-organised effort to offer prophylactic vaccination to the whole population will hold tremendous benefit for future generations of women.

- Cervical cytology is still the most important tool for secondary prevention. It should be used with enthusiasm, even in vaccinated women.

- HPV testing holds promise for the future to be used either as a primary screen or as an additional test to cytology.

\section{single suture}

\section{Asthma and air purifiers}

A randomised controlled study of purified air administered to the 'breathing zone' at night to people with allergic asthma found that active treatment over 10 weeks resulted in improved health-related quality of life compared with placebo. The novel treatment used temperature-related laminar airflow with a very low particle concentration directed to the breathing zone of sleeping teenagers and young adults with mild to moderate allergic asthma. Bronchial inflammation also improved after 5 weeks. Changes in lung function, however, were not significant.

\section{single suture}

\section{Exercise and fat accumulation}

Most studies of physical activity and obesity are usually limited by short follow-up periods and genetic selection bias, but here's a 30-year study of 16 twin pairs who were discordant for leisure time physical activity habits in 1975, and who remained so throughout the next 30 years. Magnetic resonance imaging assessed visceral, liver and intramuscular fat (the so-called high-risk fat) over time, and the authors conclude that regular physical activity seems to be an important factor in preventing high-risk fat accumulating, even after controlling for genes and childhood environment. 